\title{
SEQUELAS A LONGO PRAZO DE FRACTURAS DO CORPO E COLO DO ASTRÁGALO
}

\section{LONG-TERM RESULTS OF BODY AND NECK TALUS FRACTURES}

Ricardo Jorge Gomes de Sousa', Marta Maria Teixeira de Oliveira Massada', Manuel Alexandre Negrais Pinho Gonçalves Pereira', Isabel Maria Gonçalves Costa ${ }^{2}$, José Fernando Souzellas da Costa e Castro ${ }^{3}$

\section{RESUMO}

Objetivo: As fracturas do colo e corpo do astrágalo são lesões infrequentes. O objectivo deste estudo é avaliar a prevalência de sequelas a longo prazo. Métodos: Foi feita uma análise retrospectiva que incluiu um total de 11 doentes sujeitos a tratamento cirúrgico por fracturas do corpo ou colo do astrágalo entre Janeiro de 1997 e Dezembro de 2005. A avaliação final foi clínica (utilizando a escala AOFAS) e radiológica. Resultados: O seguimento médio foi 58,5 meses. A prevalência de lesões ósseas associadas foi de $60 \%$ (6/10). O resultado AOFAS médio foi 72 [19-100] pontos. A necrose avascular e/ou artrose pós-traumática ocorreu em metade dos doentes. A qualidade da redução cirúrgica, as fracturas do corpo e a ausência de alterações degenerativas relacionaram-se com melhores resultados funcionais. As fracturas do colo, a osteonecrose e a presença de artrose póstraumática conduziram a piores resultados. Conclusão: Há um grande potencial para sequelas tardias e compromisso funcional devido a artrose e dor crónica após esse tipo de fracturas. A redução anatómica cirúrgica é a melhor hipótese de as evitar, mas não é infalível. A taxa de necrose avascular relaciona-se com o grau de desvio inicial da fractura, mas a sua ocorrência em cada caso específico é imprevisível.

Descritores - Fraturas ósseas/complicações; Astrágalo; Osteonecrose; Osteoartrite

\begin{abstract}
Objectives: Talar neck and body fractures are unusual fractures. The purpose of this study is to determine the prevalence of long term results. Material and Methods: A retrospective analysis was carried out including 11 patients that underwent surgical treatment for body or neck talus fractures between January 1997 and December 2005. Final follow-up examination included a clinical evaluation (AOFAS score) and standard radiographs. Results: The mean follow-up time was 58.5 months. The prevalence of associated fractures was $60 \%$ (6/10). Overall AOFAS score averaged 72 [19-100]. Avascular necrosis and posttraumatic arthritis were present in half of the patients. Quality of surgical reduction, body fractures and absence of degenerative changes were correlated with better functional results. Neck fractures, osteonecrosis and posttraumatic arthritis led to inferior results. Conclusion: There is a great potential for long term functional impairment due to posttraumatic arthritis and chronic pain in this kind of fracture. Anatomic surgical reduction is the best chance to avoid them but it is not infallible. The avascular necrosis rate correlates with initial fracture displacement, but its occurrence in each specific case is unpredictable.
\end{abstract}

Keywords - Fractures, Bone/complications; Astragalus; Osteonecrosis; Osteoarthritis

\footnotetext{
1 - Interno Complementar do Serviço de Ortopedia do Hospital de Santo António, Porto, Portugal

2 - Assistente Hospitalar Graduado do Serviço de Ortopedia do Hospital de Santo António, Porto, Portugal

3 - Chefe de Serviço do Serviço de Ortopedia do Hospital de Santo António, Porto, Portugal
}

Trabalho realizado no Serviço de Ortopedia do Hospital de Santo António - Centro Hospitalar do Porto, Portugal.

Correspondência: Serviço de Ortopedia, Hospital de Santo António - Centro Hospitalar do Porto - Largo Professor Abel Salazar - 4099-001 Porto, Portugual

E-mail: ricardojgsousa@gmail.com 


\section{INTRODUÇÃO}

As fracturas do corpo e colo do astrágalo são lesões raras, mas com potencial para graves complicações a longo prazo. Historicamente, têm sido associadas a taxas de necrose avascular que variam entre $12 \%$ e $53 \%{ }^{(1-7)}$.

A artrose pós-traumática pode ser uma sequela ainda mais frequente. No entanto, a grande variabilidade nos intervalos de seguimento entre os diferentes estudos torna difícil a sua quantificação exacta. A prevalência de alterações degenerativas descritas varia entre 16 e $100 \%{ }^{(1-7)}$.

Habitualmente, essas fracturas surgem no contexto de traumatismos de alta energia e, como tal, com alta prevalência de fracturas associadas. Esse facto pode obscurecer o verdadeiro impacto da lesão astragalina no resultado clínico final. De qualquer forma, parece evidente que em grande parte dos casos será de esperar algum grau de compromisso funcional.

Decidimos avaliar em conjunto esses dois tipos de fracturas (corpo e colo), pois pensamos que partilham muitos aspectos relacionados com biomecânica, tratamento, complicações e possíveis repercussões funcionais.

O principal objectivo deste trabalho foi determinar o impacto funcional a longo prazo das fracturas do corpo e colo do astrágalo tratadas cirurgicamente no nosso hospital. Para além disso, estávamos interessados em avaliar a relação existente (ou não) entre necrose avascular, presença de alterações degenerativas articulares e o resultado clínico final. Um objectivo secundário seria descrever diferenças eventualmente existentes entre fracturas do colo e corpo.

\section{MÉTODOS}

Identificaram-se todos os doentes submetidos a cirurgia por fractura do corpo ou colo do astrágalo no nosso Hospital, num período de nove anos compreendido entre Janeiro de 1997 e Dezembro de 2005, recorrendo aos livros de registo de actividade cirúrgica. Foi feita análise retrospectiva dos respectivos processos clínicos e radiológicos.

Todos os doentes foram convocados e avaliados clinicamente utilizando a escala Kitakoa para o tornozelo e retropé da American Orthopedic Foot and Ankle Society (AOFAS), que avalia dor, função e alinhamento. Efectuou-se ainda estudo radiológico do tornozelo.

As radiografias iniciais foram avaliadas com o intuito de classificar as fracturas. Para tal foi utilizada a classificação de Hawkins modificada por Canale e Kelly ${ }^{(1)}$ para as fracturas do colo e a de $\operatorname{Marti}^{(7)}$ para as fracturas do corpo (Figura 1). A qualidade da redução obtida foi determinada através da análise de radiografias de controlo pós-operatório precoce. Para tal foram utilizados os critérios propostos por Lindvall et al ${ }^{(3)}$, que definiram redução anatómica como inexistência de desvio ou angulação da fractura, quase anatómica como existência de um desvio de 1-3mm ou angulação em varo ligeira $\left(\leq 5^{\circ}\right)$, e má redução para valores superiores (Figura 2).

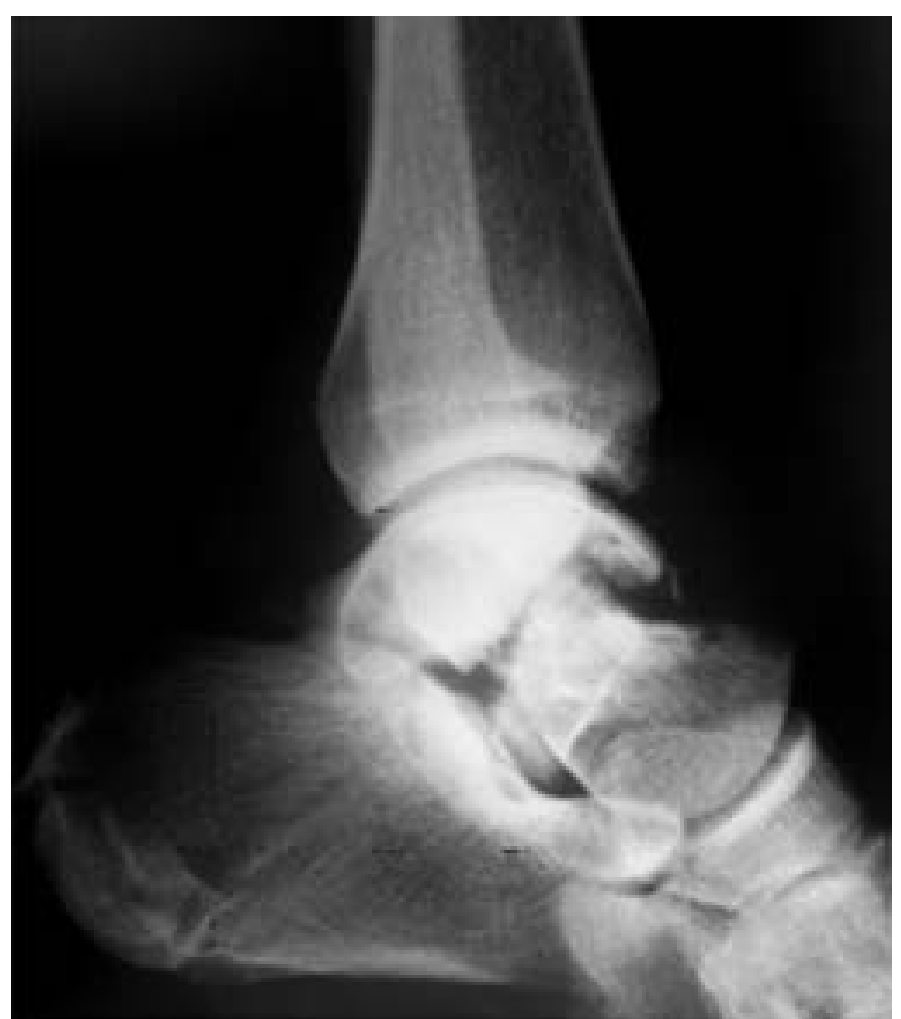

Figura 1 - Exemplo de fractura do corpo do astrálago Tipo II de Marti. Neste caso o doente apresentava fractura do calcâneo associada

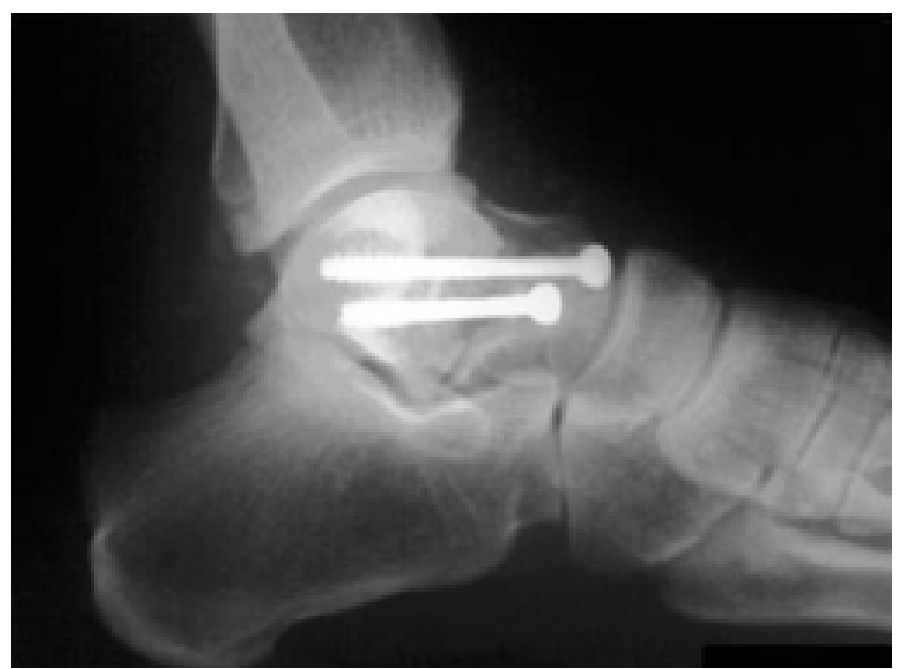

Figura 2 - Redução pós-operatória quase anatômica tipo III de Hawkins 
A presença ou não de osteonecrose teve por base a observação das radiografias pertencentes ao processo e os sinais de artrose foram pesquisados nas imagens efectuadas na consulta de revisão. Esses critérios foram determinados por um observador independente sénior (CC) que desconhecia o estado clínico de cada paciente.

Na interpretação desses resultados não foram procuradas quaisquer correlações estatísticas, pois o reduzido tamanho da amostra não permite inferências significativas.

\section{RESULTADOS}

Dos 19 doentes inicialmente identificados, apenas foi possível avaliar 11. A idade média à data do traumatismo inicial era de 38,5 [14-63] anos. Apenas um dos doentes é do sexo feminino. O tempo decorrido entre a cirurgia e a consulta de revisão foi em média de 58,5 meses, variando entre 14 meses e 10 anos.

A classificação inicial da fractura apenas foi possível em oito dos 11 doentes por falta das radiografias iniciais em três casos. Desses, quatro eram fracturas do corpo tipo II (Marti), três fracturas do colo (dois tipo II e um tipo III de Hawkins). Foi ainda encontrada uma fractura osteocondral posterior do corpo, que foi excluída do estudo por a considerarmos distinta das restantes. Os três casos em que não foi possível obter as radiografias iniciais foram ainda assim incluídos no estudo, pois o registo clínico e as radiografias pós-operatórias permitiram perceber que se tratava de fracturas do corpo $(n=1)$ ou colo $(n=2)$ do astrágalo. Foi, portanto, estudado um total de dez casos.

A alta prevalência de lesões ósseas associadas constatadas (6/10) deixa perceber a violência do traumatismo geralmente associada a esse tipo de fracturas. As mais frequentes foram a fractura do acetábulo, tíbia distal (pilão e maléolos) e calcâneo ipsilateral.

A escala utilizada (AOFAS) define Excelente resultado entre 90 e 100 pontos, Bom entre 80 e 89, Razoável entre 70 e 79 pontos e Mau resultado abaixo disso. O valor médio foi de 72 [19-100].

A osteonecrose da cúpula astragalina surgiu em cinco dos doentes (três fracturas do colo e duas fracturas do corpo). A sua presença condicionou fortemente o resultado final, com AOFAS médio de 50 pontos contra média de 93 nos casos sem necrose. Também o surgimento de artrose parece estar intimamente ligado à evolução para osteonecrose. Quatro desses cinco doentes apresentaram alterações degenerativas articulares tardias.
Cinco dos dez doentes apresentaram sinais de artrose na articulação subastragalina. Desses, apenas um apresentava atingimento isolado da subastragalina, os restantes quatro apresentavam também sinais radiológicos de artrose do tornozelo. Não se observou nenhum caso com alterações degenerativas isoladas da articulação tibioastragalina. Apenas dois dos dez doentes apresentavam artrose astrágalo-escafoideia. Não se encontraram diferenças relevantes entre a presença de artrose entre os dois subgrupos (três em cinco fracturas do colo vs dois em cinco fracturas do corpo).

A relação entre uma melhor pontuação e a ausência de alterações degenerativas foi evidente. Nos doentes com artrose o valor médio foi de 51 contra 93 nos doentes sem alterações radiográficas. Considerando apenas o critério da dor, pudemos também constatar que a sua presença se correlaciona com a existência de artrose (Figura 3).

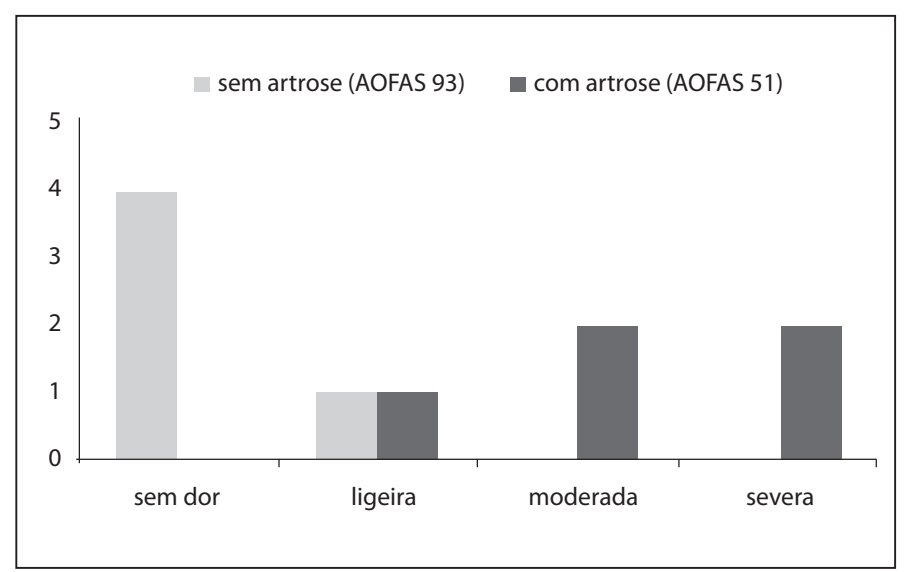

Figura 3 - Relação entre a presença de artrose e prevalência de dor

A qualidade da redução conseguida também se correlacionou positivamente com o resultado clínico e radiológico final (Tabela 1). Dos dez doentes estudados não foi possível encontrar as radiografias pós-operatórias em três deles. Dos sete restantes, quatro foram classificados como redução anatómica, dois como quase anatómica e um foi considerado como má redução.

Tabela 1 - Relação entre a qualidade da redução e resultado clínico e radiológico final

\begin{tabular}{l|c|c}
\hline \multicolumn{1}{c|}{ Qualidade da redução } & Presença de artrose & AOFAS \\
\hline Anatómica $(n=4)$ & $25 \%(1 / 4)$ & 89 \\
\hline Quase anatómica $(n=2)$ & $50 \%(1 / 2)$ & 55 \\
\hline Má redução $(n=1)$ & $100 \%(1 / 1)$ & 60 \\
\hline
\end{tabular}


Um achado curioso foi a da diferença entre os resultados das fracturas do colo (AOFAS médio: 61) e as do corpo (AOFAS médio: 82). Analisando mais pormenorizadamente os resultados, constatamos que quatro das cinco fracturas do corpo identificadas obtiveram Bom ou Excelente resultados, ao passo que três das cinco fracturas do colo foram classificadas como Mau resultado. Apenas um caso de fractura do corpo apresentava sinais de alterações degenerativas tardias.

\section{DISCUSSÃO}

\section{Impacto funcional a longo prazo}

Podemos constatar que esse tipo de fracturas pode causar um significativo impacto funcional. A média obtida (72) encontra-se no limiar inferior do Razoável. No entanto, o resultado funcional foi Excelente em quatro casos, Bom em dois e Mau nos restantes quatro doentes. Recentemente, Ebraheim et al ${ }^{(8)}$ descreveram uma proporção semelhante (nove em 16) de resultados razoáveis/maus em fracturas do corpo. Lindvall et $a l^{3}$ no seu estudo de 26 fracturas do colo ou corpo descrevem também uma grande proporção de razoáveis/maus resultados. Outros estudos, utilizando diferentes escalas de avaliação clínica que impedem a comparação directa, confirmam uma proporção que geralmente ronda os $50 \%$ de resultados não satisfatórios ${ }^{(4-6,9)}$.

\section{Osteonecrose e alterações degenerativas}

A necrose avascular da cúpula astragalina representa uma complicação específica desse tipo de fracturas e deve-se à interrupção da irrigação arterial proveniente do seio e canal do tarso ao nível do colo do astrágalo.

A sua ocorrência varia consideravelmente na literatura, mas parece evidente que se correlaciona com o grau de desvio inicial da fractura. As fracturas do colo tipo I de Hawkins desenvolvem osteonecrose em 0-24\% dos casos, as do tipo II em 0-50\% e as do tipo III e IV em $33-100 \%{ }^{(7)}$. As fracturas sem desvio do corpo (tipo I de Marti) podem evoluir para necrose avascular em 5-44\% dos casos, enquanto que ronda os $50 \%$ nas fracturas com desvio (tipo III e IV de Marti) $)^{(7)}$.

No seu relato original em $1970^{(10)}$, Hawkins descreveu um sinal radiográfico que consiste no surgimento de uma radiolucência na região subcondral da cúpula astragalina por volta da 6 a semana. Essa osteopenia traduz remodelação óssea e é altamente preditiva de revitalização óssea do corpo. Mesmo que a radiolucência seja apenas parcial (Figura 4), o prognóstico continua a ser favorável ${ }^{(11,12)}$. No entanto, tal como podemos constatar no nosso estudo, a sua ausência não implica a ocorrência de necrose avascular ${ }^{(11)}$. Podemos assim concluir que tem boa especificidade, mas baixa sensibilidade.

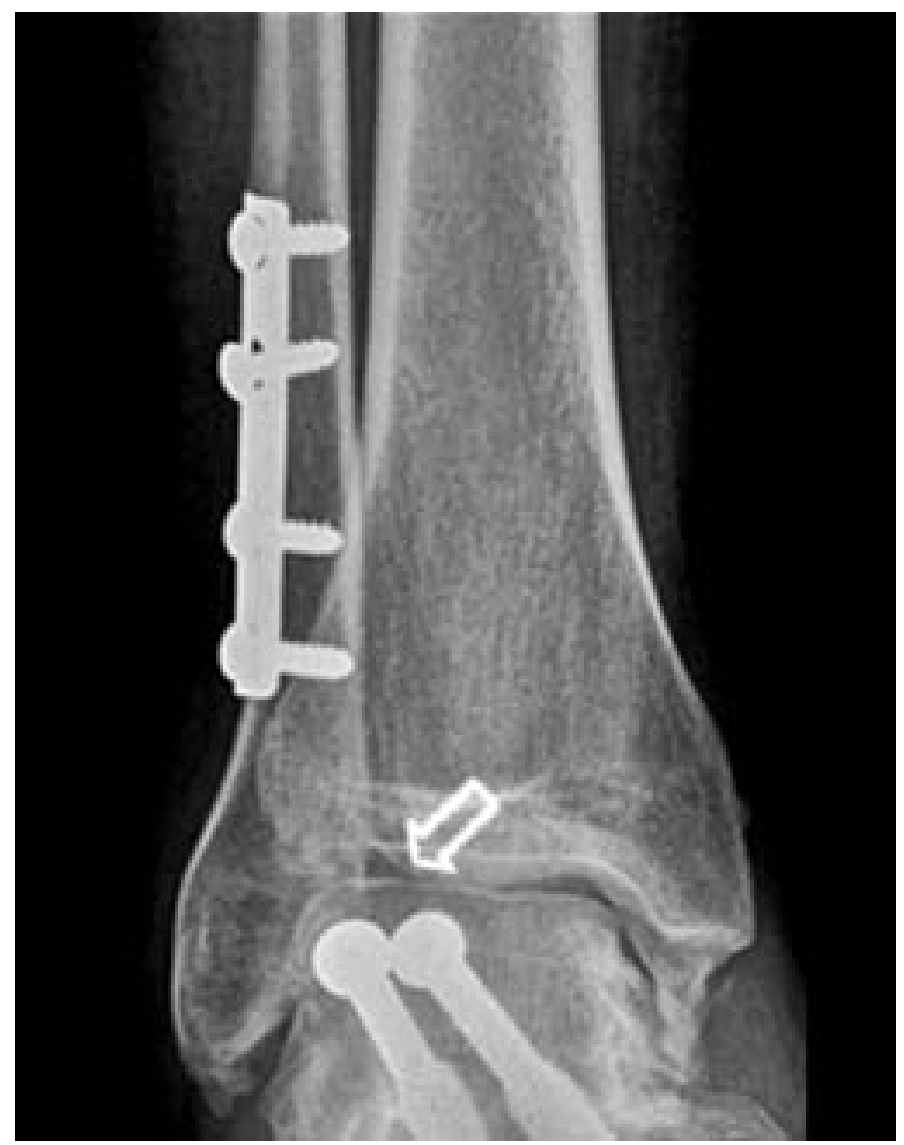

Figura 4 - Aspecto de sinal da Hawkins parcial às oito semanas de evolução

Teoricamente, o prognóstico final do tornozelo e restantes articulações adjacentes ao astrágalo parece intimamente relacionado com a extensão da eventual necrose avascular. No nosso estudo, a proporção de artrose e repercussões clínicas e funcionais foi superior nos casos em que se registou o aparecimento de osteonecrose. Porém, outras séries publicadas não revelam uma associação clara entre artrose pós-traumática e a presença de necrose avascular do astrágalo ${ }^{(3,4,7)}$.

A prevalência de artrose no nosso estudo foi de $50 \%$. A taxa expressa na literatura varia bastante (Tabela 2) e depende sobretudo do maior tempo decorrido e do critério utilizado para a definir. O estudo de Lindvall et $a l^{(3)}$ foi o único a incluir a avaliação por tomografia computorizada e isso pode justificar a taxa de $100 \%$ de alterações degenerativas subastragalinas. 
Tabela 2 - Comparação da prevalência de artrose por articulação e diferentes estudos

\begin{tabular}{l|c|c|c|c|c}
\hline & HSA & Lindvall $^{3}$ & Vallier $^{4}$ & Vallier $^{12}$ & Schulze $^{5}$ \\
\hline Ausente & $50 \%$ & $0 \%$ & $46 \%$ & $31.6 \%$ & $31.2 \%$ \\
\hline Subastragalina & $50 \%$ & $100 \%$ & - & $34.6 \%$ & $52.5 \%$ \\
\hline Subastragalina isolada & $10 \%$ & $38.5 \%$ & - & & $17.5 \%$ \\
\hline $\begin{array}{l}\text { Tornozelo isolada } \\
\begin{array}{l}\text { Subastragalina e } \\
\text { tornozelo }\end{array}\end{array}$ & $0 \%$ & $0 \%$ & - & $65.4 \%$ & $16,20 \%$ \\
\hline $\begin{array}{l}\text { Subastragalina e } \\
\text { astragalo-escafoideia }\end{array}$ & $20 \%$ & $3.8 \%$ & - & & - \\
\hline
\end{tabular}

Parece evidente que simples aparência radiológica de artrose não é condição suficiente, nem mesmo necessária para causar repercussões clínicas e/ou funcionais. Vários estudos ${ }^{(3,4,7,13,14)}$ comprovam que a presença de regiões radiopacas (indicando áreas focais de necrose) ou mesmo sinais radiológicos de artrose pós-traumática não excluem a possibilidade de um bom resultado clínico. No entanto, o atingimento total com colapso da cúpula astragalina e extensas alterações degenerativas conduz invariavelmente a piores resultados.

São muitos os factores prognósticos negativos que escapam ao controlo do cirurgião, como sendo a gravidade da lesão inicial, o desvio inicial da fractura, a extensão da lesão cartilagínea ou cominução óssea e mesmo a gravidade da lesão de partes moles ${ }^{(7)}$.

No nosso estudo, a qualidade e estabilidade da redução conseguida parecem ter sido um factor de grande importância para a obtenção de melhores resultados a longo prazo. Outros estudos chegaram à mesma conclusão ${ }^{(3,7)}$. Na ausência de luxação, o timing da fixação interna não parece afectar as taxas de necrose

\section{REFERÊNCIAS}

1. Canale ST, Kelly FB Jr. Fractures of the neck talus. Long-term evaluation of seventy-one cases. J Bone Joint Surg Am. 1978;60(2):143-56.

2. Inokuchi S, Ogawa K, Usami N, Hashimoto T. Long term follow-up of talus fractures. Orthopedics 1996;199(1):88-96.

3. Lindvall E, Haidukewych G, DiPasquale T, Herscovici D, Sanders R. Open reduction and stable fixation of isolated, displaced talar neck and body fractures. J Bone Joint Surg Am. 2004;86(10): 2229-34.

4. Vallier HA, Nork SE, Benirschke SK, Sangeorzan BJ. Talar neck fractures: results and outcomes. J Bone Joint Surg Am. 2004;86(8):1616-24.

5. Schulze W, Richter J, Russe O, Ingelfinger P, Muhr G. Surgical treatment of talus fractures - a retrospective study of 80 cases followed for 1-15 years. Acta Orthop Scand. 2002;73(3):344-51.

6. Pajenda G, Vécsei V, Reddy B, Heinz T. Treatment of talar neck fractures: clinical results of 50 patients. J Foot Ankle Surg. 2000;39(6):365-75.

7. Rammelt S, Zwipp H. Talar neck and body fractures. Injury. 2009;40(2):120-35. avascular ou o resultado final ${ }^{(3,4,13,14)}$. Assim, torna-se evidente que uma redução anatómica é a melhor hipótese de evitar sequelas tardias.

\section{Fracturas do corpo vs fracturas do colo}

Na nossa série as fracturas do corpo parecem mais "benignas" em termos de impacto funcional a longo prazo do que as fracturas do colo. Esse achado deve ser interpretado com cautela, uma vez que se trata de uma análise com poucos casos. Para além disso, as fracturas do corpo encontradas são em geral menos graves em termos de classificação do que as do colo. Lindvall et $a l^{(3)}$, numa comparação directa de fracturas do corpo e do colo, não encontraram diferença estatisticamente significativa entre pontuação AOFAS, taxas de osteonecrose ou prevalência de artrose pós-traumática.

\section{CONCLUSÃO}

Tal como a maioria dos estudos sobre este tema, o nosso tem várias limitações. A série analisada é pequena e, portanto, estatisticamente frágil. O cariz retrospectivo do estudo dificultou a obtenção de informações clínicas mais detalhadas e mesmo de algumas radiografias que teriam sido importantes.

Não obstante, o longo recuo (em média, quase cinco anos) conseguido permitiu-nos concluir que essas fracturas têm de facto um grande potencial para sequelas tardias, especialmente quando não é obtida uma redução anatómica estável.

A evolução para necrose avascular é uma das principais complicações e a sua ocorrência é de certa forma imprevisível. Os doentes devem ser devidamente informados de que a artrose pós-traumática e a dor crónica podem vir a surgir apesar do tratamento cirúrgico adequado e mesmo na ausência de sinais radiológicos de osteonecrose.

8. Ebraheim NA, Patil V, Owens C, Kandimalla Y. Clinical outcome of fractures of the talar body. Int Orthop. 2008;32(6):773-7.

9. Sanders DW, Busam M, Hattwick E, Edwards JR, McAndrew MP, Johnson KD. Functional outcomes following displaced talar neck fractures. J Orthop Trauma. 2004;18(5):265-70.

10. Hawkins LG. Fractures of the neck of the talus. J Bone Joint Surg Am. 1970;52(5):991-1002.

11. Tezval M, Dumont C, Sturmer KM. Prognostic reliability of the Hawkins sign in fractures of the talus. J Orthop Trauma. 2007;21(8):538-43.

12. Tehranzadeh J, Stuffman E, Ross SDK. Partial Hawkins sign in fractures of the talus: a report of three cases. Am J Roentgenol. 2003;181(6):1559-63.

13. Vallier HA, Nork SE, Benirschke SK, Sangeorzan BJ. Surgical treatment of talar body fracture. J Bone Joint Surg Am. 2003;85(9):1716-24.

14. Comfort TH, Behrens F, Gaither DW. Long term results of displaced talar neck fractures. Clin Orthop Relat Res;1985;(199):81-7. 